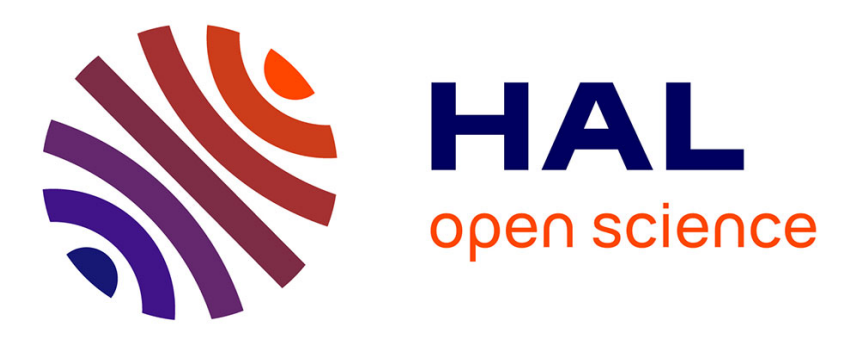

\title{
Simulating DCT and SCOT in the transport regime
}

Ugo Tricoli, Callum M. Macdonald, Turgut Durduran, Anabela Da Silva, Vadim A. Markel

\section{To cite this version:}

Ugo Tricoli, Callum M. Macdonald, Turgut Durduran, Anabela Da Silva, Vadim A. Markel. Simulating DCT and SCOT in the transport regime. OSA Biophotonics Congress: Biomedical Optics, Apr 2018, Hollywood, Floride, United States. hal-02376894

\section{HAL Id: hal-02376894 https://hal.science/hal-02376894}

Submitted on 7 Jan 2022

HAL is a multi-disciplinary open access archive for the deposit and dissemination of scientific research documents, whether they are published or not. The documents may come from teaching and research institutions in France or abroad, or from public or private research centers.
L'archive ouverte pluridisciplinaire HAL, est destinée au dépôt et à la diffusion de documents scientifiques de niveau recherche, publiés ou non, émanant des établissements d'enseignement et de recherche français ou étrangers, des laboratoires publics ou privés. 


\title{
Simulating DCT and SCOT in the transport regime
}

\author{
Ugo Tricoli $^{1, *}$, Callum M. Macdonald ${ }^{1}$, Turgut Durduran ${ }^{2}$, Anabela Da Silva ${ }^{1}$, Vadim A. \\ Markel $^{1}$ \\ ${ }^{1}$ Aix Marseille Univ, CNRS, Centrale Marseille, Institut Fresnel, F-13013 Marseille, France \\ ${ }^{2}$ ICFO-Institut de Ciències Fotòniques, The Barcelona Institute of Science and Technology, 08860 Castelldefels \\ (Barcelona), Spain. Institució Catalana de Recerca i Estudis Avançats (ICREA), 08015 Barcelona, Spain. \\ *ugo.tricoli@fresnel.fr
}

\begin{abstract}
We apply first order perturbation theory to the scalar radiative transport equation for the temporal field auto-correlation function to study DCT and SCOT sensitivity to changes in the Brownian motion of the constituent scattering particles.
\end{abstract}

OCIS codes: $110.6150,110.6955,290.4210$

\section{Introduction}

Numerous methods consider the temporal auto-correlation function in order to study the dynamical properties of a medium, e.g. DCT and SCOT. In this paper, we calculate the field correlation function in the transport regime as a solution to the CTE introduced in [1]. We show how perturbation theory can be applied to the CTE in order to calculate the sensitivity kernel relating the variation of the local Brownian motion of particles to the typical data. The Green's function of the standard radiative transport equation (RTE) can be used to construct the sensitivity kernel in the first Born approximation where the correlation time is considered to be the small parameter. We stress that the sensitivity kernel is defined for every point within the scattering medium. The sensitivity kernel is then the Jacobian matrix required in DCT or SCOT in order to perform the image reconstruction [3]. Eventually, we demonstrate how the use of the CTE instead of the diffusion approximation, can increase the contrast and resolution of reconstructions of dynamical properties of a scattering medium.

\section{Perturbative solution of the CTE}

We consider a multiply-scattering medium with moving constituent particles, which can scatter light at the working wavelength. The statistics of laser speckles in the medium can be related to the field correlation function $\Gamma(\mathbf{r}, \hat{\mathbf{s}}, \tau)$. This function has been defined in [1] in terms of a Fourier transform of the two-point correlation function for the electric field (we use different notations: $\Gamma$ instead of $G_{1}$ with the symbol $G$ being reserved for the Green's function). What is important for us here is that $\Gamma$ can be related to measurements of the speckle contrast. Note that $\Gamma$ depends on the position $\mathbf{r}$, direction of propagation $\hat{\mathbf{s}}$, and the correlation time $\tau$. In a diffusion approximation-based approach, the directional degree of freedom $\hat{\mathbf{s}}$ is averaged out. We however do not disregard the dependence of $\Gamma$ on $\hat{\mathbf{s}}$. In the continuous-wave illumination regime, $\Gamma$ obeys the stationary CTE

$$
\left(\hat{\mathbf{s}} \cdot \nabla+\mu_{t}\right) \Gamma(\mathbf{r}, \hat{\mathbf{s}}, \tau)=\mu_{s} \int \gamma^{s}\left(\mathbf{r}, \hat{\mathbf{s}}, \hat{\mathbf{s}}^{\prime}, \tau\right) A\left(\hat{\mathbf{s}}, \hat{\mathbf{s}}^{\prime}\right) \times \Gamma\left(\mathbf{r}, \hat{\mathbf{s}}^{\prime}, \tau\right) d^{2} s^{\prime}+\varepsilon(\mathbf{r}, \hat{\mathbf{s}}) .
$$

Here $\mu_{s}$ and $\mu_{t}$ are the scattering and extinction coefficients (assumed to be known), $A$ and $\varepsilon$ are the scattering phase function and the source term (the same functions that appear in the ordinary RTE), and $\gamma^{s}$ is the normalized temporal field correlation function for single scattering (defined in [1]) with the incoming and outgoing directions $\hat{\mathbf{s}}^{\prime}$ and $\hat{\mathbf{s}}$. Although the CTE is stationary, it contains explicitly the correlation time $\tau$. Moreover, this equation is somewhat different from the ordinary RTE due to the presence of the function $\gamma^{s}$. The latter is given by [1]

$$
\gamma^{s}\left(\mathbf{r}, \hat{\mathbf{s}}, \hat{\mathbf{s}}^{\prime}, \tau\right) \approx 1+\left(\hat{\mathbf{s}} \cdot \hat{\mathbf{s}}^{\prime}-1\right) 2 D_{B}(\mathbf{r}) k_{0}^{2} \tau .
$$

In this expression, $k_{0}$ is the wave number at the central frequency of the laser (assumed to be quasi-monochromatic) and $D_{B}$ is the Brownian motion diffusion constant. Obviously, this mathematical expansion requires $\tau$ to be sufficiently small. 

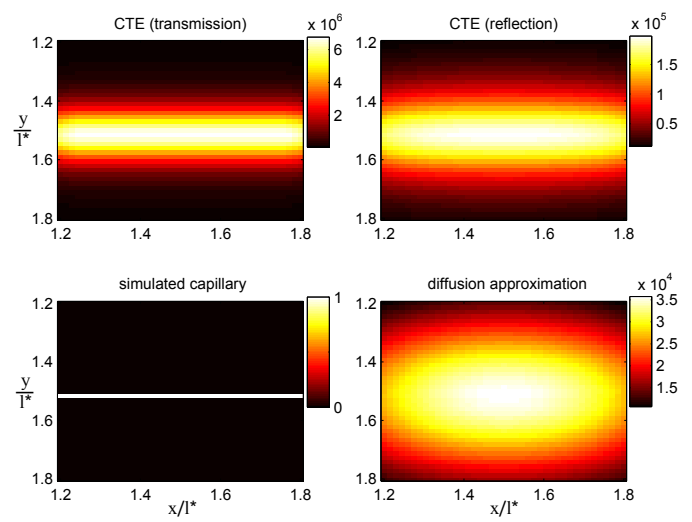

Fig. 1. Correlation function sensitivity computed via Monte Carlo simulations. The images are obtained scanning on the surface of the sample containing a tube filled with particles moving under Brownian motion with $D=0.5 \cdot 10^{-8} \mathrm{~cm}^{2} \mathrm{~s}^{-1}$. The images correspond to the shadow of the dynamically absorbing object. The result are the solution of the CTE in transmission geometry (left column) and reflection geometry (right column). The results for the diffusion approximation is in transmission geometry. The third plot shows the model object (straight capillary). The slab thickness is $1 \ell^{*}$.

Note that $D_{B}$ is a function of $\mathbf{r}$ since the medium properties can change. Applying a perturbative expansion to the CTE we arrive to the DCT sensitivity kernel (or equivalently Jacobian)

$$
K_{1}\left(\mathbf{r}_{b}, \hat{\mathbf{s}}_{b} ; \mathbf{r}_{a}, \hat{\mathbf{s}}_{a} ; \mathbf{r}\right)=\int G\left(\mathbf{r}_{b}, \hat{\mathbf{s}}_{b} ; \mathbf{r}, \hat{\mathbf{s}}\right)\left(\hat{\mathbf{s}} \cdot \hat{\mathbf{s}}^{\prime}-1\right) \times A\left(\hat{\mathbf{s}}, \hat{\mathbf{s}}^{\prime}\right) G\left(\mathbf{r}, \hat{\mathbf{s}}^{\prime} ; \mathbf{r}_{a}, \hat{\mathbf{s}}_{a}\right) d^{2} s d^{2} s^{\prime},
$$

\section{Results}

We now consider the imaging of a scattering medium in the shape of a slab. The scattering and absorption coefficients $\left(\mu_{s}=10 \mathrm{~cm}^{-1}\right.$ and $\mu_{a}=0.02 \mathrm{~cm}^{-1}$ ), and the scattering phase function (for Mie particles with asymmetry parameter $g=0.95$ and size parameter $x=7.15$ ) which corresponds to a transport mean free path $\ell^{*}=1 /\left[\mu_{a}+(1-g) \mu_{s}\right]=1.92 \mathrm{~cm}$ are constant inside the medium but the scatterer Brownian motion diffusion coefficient $D_{B}$ can vary in space. The source is a continuouswave, collimated laser beam incident at some location $\mathbf{r}_{A}$ on the surface of the slab and in the direction of the unit vector $\hat{\mathbf{s}}_{A}$. The presence of motion heterogeneities inside the slab will result in a detectable variation of the measured field correlation function. To evaluate numerically the kernel of Eq. (3) we have to expand all the terms in spherical harmonics and to reduce their product through orthogonality. Then, the reciprocity as in [2] can be applied. If a scanning strategy is adopted, an increased resolution of dynamically absorbing objects is obtained within the transport regime. This is demonstrated in Figure (1). To obtain these results, it is necessary to scan the sample with fixed relative position of the source and detector (both in the transmission and reflection geometries the source and detector are aligned).

\section{Conclusions}

The application of our method to DCT/SCOT allows the treatment of arbitrary shaped regions filled with particles undergoing Brownian motion. Moreover, arbitrary source-detector separations and orientations can be evaluated with a single simulation. This is important for DCT when many source-detector positions are used. Shortly, we believe that all these results are useful for DCT and SCOT in order to get fast simulations of arbitrary objects and source-detector positions aiming at higher resolution tomographic imaging.

\section{References}

1. D. A. Boas and A. G. Yodh, J. Opt. Soc. Am. A, 14, 1 (1997).

2. U. Tricoli, C. Macdonald, A. Da Silva and V. Markel, Optics Letters 42, 2, 362-365 (2017).

3. Hari M. Varma, Claudia P. Valdes, Anna K. Kristoffersen, Joseph P. Culver and Turgut Durduran, Biomed. Opt. Express 5, 4 (2014). 